\title{
Implementing standard antenatal care interventions: health system cost at primary health facilities in Tanzania
}

\author{
Amisa Tindamanyile Chamani ${ }^{1,2^{*}} \mathbb{D}$, Amani Thomas Mori ${ }^{1,3}$ and Bjarne Robberstad ${ }^{1}$
}

\begin{abstract}
Background: Since 2002, Tanzania has been implementing the focused Antenatal Care (ANC) model that recommended four antenatal care visits. In 2016, the World Health Organization (WHO) reintroduced the standard ANC model with more interventions including a minimum of eight contacts. However, cost-implications of these changes to the health system are unknown, particularly in countries like Tanzania, that failed to optimally implement the simpler focused ANC model. We compared the health system cost of providing ANC under the focused and the standard models at primary health facilities in Tanzania.

Methods: We used a micro-costing approach to identify and quantify resources used to implement the focused ANC model at six primary health facilities in Tanzania from July 2018 to June 2019. We also used the standard ANC implementation manual to identify and quantify additional resources required. We used basic salary and allowances to value personnel time while the Medical Store Department price catalogue and local market prices were used for other resources. Costs were collected in Tanzanian shillings and converted to 2018 US\$.

Results: The health system cost of providing ANC services at six facilities ( 2 health centres and 4 dispensaries) was US\$185,282 under the focused model. We estimated that the cost would increase by about $90 \%$ at health centres and $97 \%$ at dispensaries to US\$358,290 by introducing the standard model. Personnel cost accounted for more than one third of the total cost, and more than two additional nurses are required per facility for the standard model. The costs per pregnancy increased from about US\$33 to US\$63 at health centres and from about US\$37 to US\$72 at dispensaries.

Conclusion: Introduction of a standard ANC model at primary health facilities in Tanzania may double resources requirement compared to current practice. Resources availability has been one of the challenges to effective implementation of the current focused ANC model. More research is required, to consider whether the additional costs are reasonable compared to the additional value for maternal and child health.
\end{abstract}

Keywords: Cost, Antenatal care, Health system, Primary health care, Tanzania

*Correspondence: tindamanyilea@gmail.com

${ }^{1}$ Department of Global Public Health and Primary Care, Section for Ethics and Health Economics, University of Bergen, Bergen, Norway

Full list of author information is available at the end of the article

\section{Introduction}

In 2016, the World Health Organization (WHO) changed its Antenatal Care (ANC) guideline from a focused ANC model [1] to a standard ANC model, to reduce perinatal mortality and improve women's experience of care. The standard model has 49 recommendations, which are grouped into five types of interventions. Compared to the focused model, the 
new model adds calcium supplementation throughout pregnancy to reduce the risk of pre-eclampsia (nutritional intervention), systematic screening for active tuberculosis and early ultra-sounding (maternal and fetal assessment intervention), up to 6 month dosing with sulfadoxine-pyrimethamine (SP) for preventing malaria, 7 day antibiotic regimen for asymptomatic bacteriuria (preventive measures intervention) and a minimum of eight contacts with a skilled health personnel to reduce perinatal mortality and improve women's experience of care (health systems intervention) $[2,3]$. Despite the expected benefits, there are financial concerns in developing countries, particularly in countries that did not effectively implement the simpler focused model [4-6]. Implementation of a more resource demanding standard model, need to be considered in the light of resource scarcity and other competing priorities.

The focused model recommends four visits for a normal pregnancy, i.e., one visit in each first and second trimesters and two visits in the third trimester. In this model, recommendations fall within screening, receiving therapeutic interventions and health education. The implementation manual suggests the availability of all services at the ANC unit, including rapid and easy to perform tests. It also suggests that, 30 to $40 \mathrm{~min}$ of personnel time should be used for the first visit, while an activity time of 20 min should be used for each of the subsequent three visits [1].Pregnant women with special needs follows an advanced version but are eligible to the basic one afterwards.

Tanzania adapted the focused ANC model in 2002, and is still using it to screen, provide therapeutic interventions and educate pregnant women [7]. Screening for malaria at the first ANC visit is an additional recommendation. The majority of pregnant women receive care from nurses and midwives at primary health facilities (dispensaries and health centers), but are referred to hospitals when advanced care is needed [7, 8]. At primary health facilities, a group health education session is normally offered in the morning, followed by individualized assessment, screening, counselling, and other interventions. During screening, some tests are conducted by nurses within the ANC unit while additional tests are performed by laboratory technicians at the facility laboratory.

Although Tanzania has documented improvement in focused ANC implementation such that $98 \%$ of pregnant women visit ANC at least once and 51\% manage to complete four visits [9], there are remaining challenges. For example, only $24 \%$ of all pregnant women go for their first visit before the 4th month of pregnancy, and there are reports of underutilization, inadequate service provision, poor quality of care and scares resources $[4,7,9-15]$.

Few studies have documented resources used for ANC in Tanzania and none have considered the costs of implementing the standard ANC model. Von Both (2008) estimated consultation costs from the health system perspective to be US $\$ 2.5$ per visit [16], while Kowalewski (2002) estimated indirect user cost of US $\$ 9.9$ per visit at primary health facilities [17]. A study by Saronga (2014) in rural areas of southern Tanzania, reported a cost of US\$16.4 per visit from a health system perspective [18]. In 2015, a study in the neighboring country Rwanda estimated a cost of US $\$ 10.65$ per visit [19] under the focused model and US\$9.9 per visit after addition of some recommendations for the standard model [20], both from a health system perspective.

While the WHO's recommendations influence guideline updates in developing countries, country-level economic evidence to support such decisions are scare. Therefore, our aim was to estimate the cost of providing ANC services at primary health facilities in Tanzania under two scenarios: (1) the current practice, which reflects a suboptimal implementation of the focused ANC model, and (2) a hypothetical full implementation of new recommendations from the standard ANC model.

\section{Methodology \\ Study settings}

We conducted this study in Kigamboni and Korogwe districts. Kigamboni is situated along the Indian Ocean, south-east of Dar es Salaam (the largest city) and is divided into nine wards with a population of 206,000 [21]. Kigamboni has a total of 21 public health facilities, including one designated hospital, two health centres and 18 dispensaries. Korogwe district is within Tanga region, $283 \mathrm{~km}$ north of Dar es Salaam and has a population of about 260,000. Korogwe district provides health services from one district hospital, three public health centers and 44 dispensaries.

From each district, we purposively chose three primary health facilities i.e., one health centre and two dispensaries. We selected facilities which attended many pregnant women for a year, to estimate maximum resources used for the focused model, and maximum resources required for the standard model. Both dispensaries and health centres provide all basic ANC services in Tanzania. A dispensary is the lowest post and offers mainly outpatient services, while a health center provides more services and to a larger population. 


\section{Data collection}

We collected data from a health system perspective using a micro-costing or bottom-up approach. This perspective excludes patients costs of accessing health care such as transportation, cost-sharing, or time off work. Data collection involved documentation review, physical inventory, and interviews with healthcare workers. We identified actual resources used to provide focused ANC for one financial year, from July 2018 to June 2019. For the standard ANC model, we used an implementation manual to model additional resource that would be required, since the intervention is not yet implemented in Tanzania and observational data is consequently unavailable. We classified resources consumed within 1 year as recurrent cost; these included personnel, non-medical and medical supplies, medicines, laboratory supplies, utility, maintenance, and repair of capital items. We categorized resources that last more than a year as capital cost items, which included buildings, equipment, furniture and national level programme cost [22].

\section{Quantification and valuation of resources Personnel}

To cost the focused ANC, we interviewed each involved health personnel identified, about his/her qualifications, salary scale, allowances, and a proportion of their working time devoted to ANC compared to other duties. We assumed that pregnancies are evenly distributed over the year and that time allocated to ANC did not vary over the study period. We included gross salary, overtime and uniform allowances when calculating personnel costs. We excluded annual leave cost because data were unreliable. We used gross salary rates for a medical attendant to value the effort of skilled and trained volunteers working as medical attendants. For each personnel, we multiplied the proportion of time allocated to ANC with the annual cost of a full position to estimate personnel cost attributed to ANC. For non-medical personnel (guards and cleaners), we used the proportional floor space for ANC activities as allocation factor.

The WHO did not recommend activity time for the standard ANC model as they did for the focused model. Likewise, there is no recommended time for administrative duties related to ANC under the focused model. We assumed that the time allocated to ANC by nurses and laboratory personnel was the total of activity time and the time for administrative duties. We calculated a ratio between the allocated time (calculated above) and the activity time (suggested by the WHO) of $40 \mathrm{~min}$ (first visit) and $20 \mathrm{~min}$ (subsequent visits). This ratio indicated the proportion of allocated time that was used for administrative duties, assuming the activity time used resembled the suggestion by the WHO. To calculate personnel time for the standard model, we first assumed that the activity time use per visit for focused ANC would apply also to the standard model. We then used the ratio (calculated above) to factor in the time that will be used for administrative duties. With these inputs, we could derive additional personnel time required to switch from focused to standard ANC for each of the included facilities and per pregnancy. We used the annual cost for nurses at each facility to value the additional time use of introducing standard ANC. For other personnel we assumed that time allocated to ANC will not change with a new model. To account for a new routine ultrasound scan we included the annual cost of a sonographer at each facility and assumed 50\% allocation to ANC.

\section{Supplies}

We used ANC monthly reports to quantify medicine and laboratory supplies consumed. Monthly reports record types and numbers of tests performed, vaccines provided, and medicines administered. For example, with a report of 260 doses of Tetanus Toxoid vaccines, we estimated 13 vials of the vaccine (one vial contains $10 \mathrm{mls}$ and the standard dose is $0.5 \mathrm{mls}$ ) and 260 syringes of $1 \mathrm{ml}$ each. We used the standard ANC recommendations, shown in Table 1, to quantify additional supplies, including calcium supplements for 6 months and seven days antibiotic for asymptomatic bacteriuria. We assumed that $13 \%$ of pregnant women have asymptomatic bacteriuria in Tanzania [23] and therefore would receive treatment. We quantified other medical supplies (gloves, cotton wool, spirit, bedsheet, and safety boxes) issued to ANC and the laboratory from pharmacy registers, and additional supplies for a standard model based on the recommendations. The allocation of shared medical supplies reflected nurses and laboratory staff time allocation to ANC. We also included thermal and tissue papers and ultrasound jelly for a routine scan.

We interviewed either a facility accountant or facility in-charge on expenses incurred on cleaning detergents and equipment (bucket, broom, moppers) and allocated them using the same factor as for cleaners (see above). We quantified stationaries used at the laboratory and ANC, and we assumed that consumption would double under the standard ANC model. We increased the total quantity for each recurrent supply by $5 \%$ for spoilage, which is common in applied costing studies [24]. We used the Medical Store Department (MSD) price catalogue (2018/2019) and local market prices to identify values. These costs were further adjusted upward by $10 \%$ to account for local transport from the supplier [24]. The annual cost was estimated as the product of adjusted quantities, adjusted unit cost and allocation to ANC for each supply. 
Table 1 A comparison of recommendations between the focused and the standard antenatal care models

\begin{tabular}{llc}
\hline Output per pregnancy & Focused ANC (Tanzania) & $\begin{array}{c}\text { Standard } \\
\text { ANC }\end{array}$ \\
\hline Number of visits & 4 & 8 \\
Anemia test by hemoglobinometer & 4 & 3 \\
Urine dipstick test & 4 & 3 \\
Blood grouping test & 1 & 1 \\
Malaria by rapid diagnostic test (mRDT) & 1 & 1 \\
Syphilis test & 2 & 2 \\
HIV test & 2 & 2 \\
Tetanus Toxoid vaccine & Depend on the previous vaccination status \\
Seven-day antibiotic for asymptomatic bacteriuria & 0 & $13 \%$ of the \\
& & population \\
Monthly Sulfadoxine Pyrimethamine for malaria & $\geq 3$ & 6 \\
Long-Lasting Insecticide Treated Net (LLITN) & 1 & 1 \\
Ferrous and folic acid supplements(months) & $\geq 3$ & 6 \\
Antihelminthes (Albendazole 400 mg) & 1 & 1 \\
Calcium supplementation (months) & 0 & 6 \\
Routine ultrasound scan & 0 & 1 \\
\hline
\end{tabular}

\section{Water, gas, and electricity}

We interviewed the facility accountant or facility-in charge about water, gas, and electricity bills. We allocated $50 \%$ of the gas cost (used to store vaccines during electricity cuts of) to ANC because the freezer also stores vaccines used for other programmes. We used the proportion of equipment and rooms for ANC unit to allocate electricity and water bills for the focused model. For the standard model, we also adopted the electricity bill from a facility installed with an air condition at ANC unit to other facilities, to cater for air conditioning the ultrasound room.

\section{Capital items}

We used the MSD price catalogue or local market prices to assign values to identified equipment and furniture. The proportion of time allocated to ANC by personnel involved with specific equipment and furniture was used as the allocation key. For the standard ANC model, we in addition added annual costs for the ultrasound machine, examination bed, movable chair, washbasin stand, double step, and air-condition. These were allocated to ANC by $50 \%$, an assumption made in the WHO implementation manual for standard ANC [2]. Assumptions about useful life years for capital items were adopted from the WHOs "Choosing Interventions that are Cost-Effective project" [25], and in addition we assumed 10 years for the ultrasound machine. We calculated annual costs using a $5 \%$ interest rate [22].

We used a tape measure to estimate total floor space of each facility and subsequently allocated it to ANC using personnel time. We adapted the costs used to construct a Reproductive and Child Health $(\mathrm{RCH})$ building in Korogwe in 2008, assuming 20 years of useful life and $5 \%$ interest rate. Observations made during data collection support the idea that the standard model can be facilitated within buildings already available, with minor renovations for the ultrasound machine and its security. Finally, we added $5 \%$ of the total capital cost for maintenance and repair and then $10 \%$ of the total annual cost at facility level represented national level programme cost [26].

\section{Data analysis}

We adapted the data collection tools from the Costing Guidelines for HIV Prevention Strategies [27], and used Microsoft Excel $^{\circledR}$ for compilation and analysis. We calculated the total cost by aggregating annual cost of all supplies, personnel, and capital goods. We calculated the unit cost per visit as the ratio of total costs to the number of ANC visits, and the cost per pregnancy as the ratio of total cost to the number of first visits. Items were valued in TSh and converted to US\$ using the Bank of Tanzania exchange rate for early July 2018 (US $\$ 1=$ TSh2278) [28].

\section{Results \\ ANC characteristics of study facilities}

We recorded a total of 19,342 visits across six primary health facilities, it is $90 \%(19,342 / 21,080)$ of total visits expected with full compliance of the focused model (Table 2). Pregnant women at a health centre in Kigamboni (health centre ${ }_{1}$ ) attended more than the recommended four visits. Possibly some women were 
Table 2 Number of Antenatal care visits recorded and estimated at each study facility

\begin{tabular}{lllll}
\hline & \multicolumn{2}{l}{ Focused ANC } & & Standard ANC $^{\mathbf{b}}$ \\
\cline { 2 - 4 } Health facility & First visits & Annual visits & Visit/ pregnancy & Annual visits \\
\hline $\begin{array}{l}\text { Health centres } \\
\text { Health centre }\end{array}$ & 1,676 & 7714 & 4.6 & 13,408 \\
Health centre & 742 & 2149 & 2.9 & 5936 \\
Dispensaries & & & 3.4 & 6360 \\
Dispensary & 795 & 2735 & 3.8 & 8152 \\
Dispensary & 1019 & 3886 & 3 & 3176 \\
Dispensary & 397 & 1140 & 2.7 & 5128 \\
Dispensary & 641 & 1718 & & 42,160 \\
All facilities & 5270 & 19,342 & \\
\hline
\end{tabular}

${ }^{a}$ Observed actual numbers

${ }^{\mathrm{b}}$ Estimated assuming 8 visits per pregnancy

referred from dispensaries for specialized care including ultrasound.

\section{Personnel time}

Table 3 presents the observed time (in hours) allocated to focused ANC by nurses and laboratory staff at the six health facilities under the focused model and additional time required for the standard model. The ratio between allocated time and activity-time for the focused model suggests that time use for personnel was 1.6 to 3.8 times higher compared to what was suggested by the WHO. At health centre ${ }_{1}$ with a ratio of 1.66 , the intervals between one attendance and the other were $66 \mathrm{~min}$ for a first visit and $33 \mathrm{~min}$ for a revisit. While at dispensary ${ }_{1}$ with a ratio of 3.75, the intervals were $2 \mathrm{~h}$ and $30 \mathrm{~min}$ for a first visit and $75 \mathrm{~min}$ for a revisit. Within current levels of personnel productivity, facilities need between 1,145 and $4535 \mathrm{~h}$ of personnel per year to implement standard ANC model, equivalent to about one and three additional nurses. Each pregnant woman will require between 1.8 and 5.7 additional hours of personnel time throughout pregnancy.

\section{Annual cost and unit cost}

The detailed costing for recurrent and capital items under the focused and the standard models are shown in the Additional file 1. Health facilities in Kigamboni (Health centre $_{1}$, dispensary 1and $2_{2}$ ) recorded more visits and higher cost compared to health facilities in Korogwe (Health centre $_{2}$ Dispensaries $_{3 \text { and }}$ ). The results in Table 4 suggest that the cost will increase from US $\$ 30,880$ to US $\$ 59,715$ with implementation of a standard model, at each primary health care facility recording more than 1000 ANC visit per year. More specifically, the cost will increase from US\$40,172 to US $\$ 75,898$ at health centres and from US\$26,234 to US\$51,263 at dispensaries. The unit cost per pregnancy will increase from about US\$33 to about US\$63 at health centres, and from about US\$37 to US\$72 at dispensaries. The unit cost per visit will not change substantially with guideline update, the increased costs are largely attributable to increased number of visits.

The costs for all cost categories increased relatively proportional when moving from a focused to a standard ANC model. For both models, personnel cost was the most important accounting for a third and above of the

Table 3 Time (hours) used for the focused model and time required for the standard model

\begin{tabular}{|c|c|c|c|c|c|c|}
\hline & \multicolumn{2}{|c|}{ Health center ( 2 ) } & \multicolumn{4}{|c|}{ Dispensaries (4) } \\
\hline & $\mathrm{HC}_{1}$ & $\mathrm{HC}_{2}$ & Dispensary $_{1}$ & Dispensary $_{2}$ & Dispensary $_{3}$ & Dispensary $_{4}$ \\
\hline A. Allocated personnel time for focused ANC & 5184 & 2112 & 4416 & 3744 & 864 & 1248 \\
\hline B. Recommended Activity-time 1 -focused ANC & 3130 & 964 & 1177 & 1635 & 512 & 786 \\
\hline C. Ratio (A/B) & 1.66 & 2.19 & 3.75 & 2.29 & 1.69 & 1.59 \\
\hline D. Activity time ${ }_{2}$-standard-ANC & 5028 & 2226 & 2385 & 3057 & 1191 & 1923 \\
\hline E. Personnel time for standard ANC ( $\left.C^{*} D\right)$ & 8328 & 4879 & 8951 & 7000 & 2009 & 3052 \\
\hline F. Additional time (E-A) & 3144 & 2767 & 4535 & 3256 & 1145 & 1804 \\
\hline G. Additional time per pregnancy & 1.88 & 3.73 & 5.70 & 3.20 & 2.88 & 2.81 \\
\hline
\end{tabular}


Table 4 A comparison of antenatal care cost between focused and standard antenatal care models at health centres and dispensaries in Tanzania

\begin{tabular}{|c|c|c|c|c|c|c|}
\hline \multirow[t]{2}{*}{ Cost in US\$ } & \multicolumn{2}{|c|}{ Health centres (2) } & \multicolumn{2}{|c|}{ Dispensaries (4) } & \multicolumn{2}{|l|}{ All facilities (6) } \\
\hline & Focused ANC & Standard ANC & Focused ANC & Standard ANC & Focused ANC & Standard ANC \\
\hline Personnel & $30,483(38 \%)$ & $50,591(33 \%)$ & $38,116(36 \%)$ & $73,186(35 \%)$ & $68,599(37 \%)$ & $123,777(35 \%)$ \\
\hline Medicine/medical supplies & $21,846(27 \%)$ & $49,206(32 \%)$ & $25,441(24 \%)$ & $59,746(29 \%)$ & $47,287(26 \%)$ & $108,952(30 \%)$ \\
\hline Laboratory supplies & $11,076(14 \%)$ & $24,649(16 \%)$ & $15,744(15 \%)$ & $30,028(15 \%)$ & $26,821(14 \%)$ & $54,676(15 \%)$ \\
\hline Other recurrent items & $2,146(3 \%)$ & $4,449(3 \%)$ & $3,978(4 \%)$ & $9,172(4 \%)$ & $6,124(3 \%)$ & $13,621(4 \%)$ \\
\hline Capital items & $14,793(18 \%)$ & $22,901(15 \%)$ & $21,658(21 \%)$ & $34,363(17 \%)$ & $36,451(20 \%)$ & $57,264(16 \%)$ \\
\hline Total cost & 80,344 & 151,797 & 104,938 & 206,493 & 185,282 & 358,290 \\
\hline Cost per facility & 40,172 & 75,898 & 26,234 & 51,623 & 30,880 & 59,715 \\
\hline Cost per ANC visit & 8.1 & 7.8 & 11.1 & 9.1 & 9.6 & 8.5 \\
\hline Cost per pregnancy & 33.2 & 62.8 & 36.8 & 72.4 & 35.2 & 68 \\
\hline
\end{tabular}

total cost, followed by medicine and medical supplies, capital items, laboratory supplies and other recurrent items. Additional personnel time and the cost of a sonographer increased annual personnel cost. Calcium supplementation for 6 months (US\$22,445 across facilities), up to six doses of SP, seven-day antibiotics to $13 \%$ of the population and other medical supplies, doubled medicine and medical supplies cost.

\section{Discussion}

Our findings show that, more resources are required to implement the standard ANC model at primary health facilities in Tanzania. At each health centre with more than 2000 ANC visits annually, two additional nurses will be required, while one to three additional nurses will be required at dispensaries with more than 1000 annual visits. Also, the resources required for medical and laboratory supplies would need to double. It is important to emphasize that results for the standard ANC model were extrapolations built on facility data.

The difference between unit cost per visit at a health centre (US\$8.1) and a dispensary (US\$11.1) was small in our study. This contrasts with estimates from Mtwara in South of Tanzania, where unit costs were substantially higher at health centres (US\$19) than at dispensaries (US\$ 4.75) [18]. On the other side, our estimates are relatively similar to a study from Rwanda, where unit costs for the focused model were estimated to be US\$ 10.65 and US $\$ 10$ for the standard model $[19,20]$.

Personnel time was the main cost driver for both ANC models, and represented more than one-third of the total cost at the health centre and dispensary. Our result shows allocated time exceeded activity time differently at each facility, including time for administrative duties. A simulation study in Tanzania estimated the activity time of 46 min and 36 min for counselling and few test at ANC for the first visit and subsequent visits respectively [29], while observational studies have reported the activity time of less than $20 \mathrm{~min}$ for each visit [30]. Our analysis was based on self-reporting, and it was not possible to differentiate the activity time and the time for administrative duties, which would have required a time sequence study. But we see the potential to improve personnel productivity at some facilities, which could reduce personnel cost for the standard model if appropriately addressed. This argument is based on our data that shows large variance across facilities in the ratio between allocated time and activity time across facilities.

Resources required for medicine, medical and laboratory supplies will be more than two times for both health centres and dispensaries with implementation of standard ANC. Calcium supplementation is one of the drivers, despite that it was not cost effective in Ethiopia [31]. Our data indicate that, coverage for medicines and laboratory tests was still not optimal seventeen years after adoption of the focused model. Coverage was lower at facilities in Korogwe (health centre ${ }_{2}$ Dispensary ${ }_{3}$ and 4 ) compared to facilities in Kigamboni (health centre ${ }_{1}$ Dispensary ${ }_{1}$ and 2) (Additional File 1). The stock status has been one of the challenges to effective coverage of the focused ANC recommendation. Therefore, economic evaluation and implementation studies of new strategies to ensure availability of supplies are important before adoption of a more resourceful model.

Our estimation of unit costs for the standard model assumes that number of pregnancies will not change. Our costing included resources required to provide ultrasound under the standard model. It is not clear if ultrasound use will improve fetal and maternal outcomes [32], but it is possible that standard ANC will improve adherence, which subsequently could improve health outcomes and reduce unit costs per visit. This study was 
undertaken in facilities which attended many pregnant women annually. For smaller health facilities, the unit cost of ANC per pregnancy could be somewhat higher.

\section{Study limitations}

This study has several limitations, first, we purposively sampled six relatively large primary health facilities attending many pregnant women. The plan was to estimate the highest possible annual cost, which can be used by policy makers when budgeting for the standard ANC model. However, the approach also limits the generalizability of our findings in Tanzania, especially to facilities attending few pregnant women. We also estimated resources for an ambition to fully implement the standard ANC model. Second, incomplete, or inaccurate facility records compelled us to exclude some information, such as the cost of managing sick pregnant women, which represents potential underreporting. Third, we used self-reporting to allocate personnel time to antenatal care which is prone to information bias. This could have resulted into overestimation or underestimation of personnel cost. Nevertheless, personnel were asked to allocate time to each of their responsibilities, which might have reduced the bias. Finally, we considered cost from a provider's perspective, while ignoring the additional cost imposed on pregnant women from more facility visits. This may underestimate the overall societal cost implication of policy change from focused to standard antenatal care.

\section{Conclusion}

The introduction of standard ANC in primary health facilities in Tanzania may double the resources requirements compared to current practice under the assumption that all resources are being efficiently used. While resource availability has been a challenge for effective implementation of the focused ANC model, more research is required, to consider whether the cost of implementing standard ANC model are reasonable compared to the additional value for maternal and child health.

\section{Abbreviations}

ANC: Antenatal care; WHO: World Health Organization.

\section{Supplementary Information}

The online version contains supplementary material available at https://doi. org/10.1186/s12962-021-00325-0.

Additional file 1. Annual costs for recurrent and capital items under the focused and the standard ANC models.
Acknowledgements

Not applicable

\section{Authors' contributions}

ATC, ATM and BR contributed to the development of the concept and study design. ATC collected, analysed and interpreted the data, and wrote the first draft of the manuscript. ATM and BR contributed to the analysis and interpretation of the results. All authors revised the manuscript critically and approved the final version. All authors have read and approved the final manuscript.

\section{Funding}

This study is part of the project NORPART-2016/10480, which aims to strengthen health economics research capacity between the University of Bergen-Norway and Muhimbili University of Health and Allied Sciences Tanzania with funding from the Norwegian Agency for International Cooperation and Quality Enhancement in Higher Education. The funder played no role in the design of the study, the data collection, analysis, or interpretation of the results, or the writing of the manuscript.

\section{Availability of data and materials}

All data generated or analysed during this study are included in this published article.

\section{Declarations}

\section{Ethics approval and consent to participate}

We obtained approval from the Ethical Review Committee of the Tanzania National Institute for Medical Research (NIMR/HQ/R.8a/Nol.IX/3139) and the exemption from Regional Ethics Committee, Western Norway (2019/758/REK Vest) as our study was deemed outside the scope of the Norwegian health research act. We also obtained written permissions from the Office of the President of the Regional Administration and Local Government; Kigamboni Municipal and Korogwe District Directors, and District Medical Officers. We sought verbal consent before the interview with health care providers.

\section{Consent for publication}

Not applicable.

\section{Competing interests}

All authors declare no competing interests.

\section{Author details}

'Department of Global Public Health and Primary Care, Section for Ethics and Health Economics, University of Bergen, Bergen, Norway. ${ }^{2}$ Department of Pharmaceutics and Pharmacy Practice, School of Pharmacy, Muhimbili University of Health and Allied Sciences, Dar es Salaam, Tanzania. ${ }^{3} \mathrm{Chr}$ Michelsen Institute, Bergen, Norway.

Received: 6 April 2021 Accepted: 14 October 2021

Published online: 07 December 2021

\section{References}

1. World Health Organization. Antenatal Care Randomized Trial Manual for the implementation of the New Model. Geneva: World health organization; 2002.

2. World Health Organization. Recommendations on antenatal care for a positive pregnancy experience. Geneva: World Health Organization; 2016. Report No.: 9241549912.

3. Downe S, Finlayson K, Tuncalp O, Metin Gulmezoglu A. What matters to women: a systematic scoping review to identify the processes and outcomes of antenatal care provision that are important to healthy pregnant women. BJOG Int J Obstet Gynaecol. 2016;123(4):529-39.

4. Konje ET, Magoma MTN, Hatfield J, Kuhn S, Sauve RS, Dewey DM. Missed opportunities in antenatal care for improving the health of pregnant women and newborns in Geita district, Northwest Tanzania. BMC Pregnancy Childbirth. 2018;18(1):394. 
5. Morón-Duarte LS, Ramirez Varela A, Segura O, Freitas da Silveira M. Quality assessment indicators in antenatal care worldwide: a systematic review. Int J Qual Health Care. 2018. https://doi.org/10.1093/intqhc/mzy206.

6. Weeks A, Temmerman M. New WHO antenatal care model—quality worth paying for? Lancet. 2016;388(2574):5.

7. Kearns A, Hurst T, Caglia J, Langer A. Focused antenatal care in Tanzania: delivering individualised, targeted, high-quality care. Maternal health task force report; 2014. www.maternalhealthtaskforce.org.

8. The United Republic of Tanzania Ministry of Health and Social welfare Focused antenatal care, malaria and syphilis in pregnancy: orientation package for service providers; 2009. https://pdf.usaid.gov/pdf_docs/ pnaea268.pdf.

9. Ministry of Health Tanzania Mainland, Ministry of Health Zanzibar, National Bureau of Statistics, Office of the Chief Government Statistician, ICF. Tanzania Demographic and Health Survey and Malaria Indicator Survey (TDHS-MIS) 2015-16 Dar es Salaam, Tanzania, and Rockville, Maryland, USA; 2016. https://dhsprogram.com/pubs/pdf/fr321/fr321.pdf. Accessed 17 Nov 2019.

10. Solnes Miltenburg A, van der Eem L, Nyanza EC, van Pelt S, Ndaki P, Basinda N, et al. Antenatal care and opportunities for quality improvement of service provision in resource limited settings: a mixed methods study. PloS One. 2017;12(12): e0188279.

11. Afnan-Holmes $\mathrm{H}$, Magoma M, John T, Levira F, Msemo G, Armstrong CE, et al. Tanzania's countdown to 2015: an analysis of two decades of progress and gaps for reproductive, maternal, newborn, and child health, to inform priorities for post-2015. Lancet Glob Health. 2015;3(7):e396-409.

12. Hodgins S, D'Agostino A. The quality-coverage gap in antenatal care: toward better measurement of effective coverage. Global Health Sci Pract. 2014;2(2):173-81.

13. Benova L, Tunçalp Ö, Moran AC, Campbell OMR. Not just a number: examining coverage and content of antenatal care in low-income and middle-income countries. BMJ Global Health. 2018. https://doi.org/10. 1136/bmjgh-2018-000779.

14. Owili PO, Muga MA, Mendez BR, Chen B. Quality of care in six subSaharan Africa countries: a provider-based study on adherence to WHO's antenatal care guideline. Int J Qual Health Care. 2018. https://doi.org/10. 1093/intahc/mzy105.

15. Conrad P, Schmid G, Tientrebeogo J, Moses A, Kirenga S, Neuhann F, et al. Compliance with focused antenatal care services: do health workers in rural Burkina Faso, Uganda and Tanzania perform all ANC procedures? Trop Med Int Health. 2012;17(3):300-7.

16. von Both C, Jahn A, Fleßa S. Costing maternal health services in South Tanzania: a case study from Mtwara Urban District. Eur J Health Econ. 2008;9(2):103-15.

17. Kowalewski M, Mujinja P, Jahn A. Can mothers afford maternal health care costs? User costs of maternity services in rural Tanzania. Afr J Reprod Health. 2002;6(1):65-73.

18. Saronga HP, Duysburgh E, Massawe S, Dalaba MA, Savadogo G, Tonchev $P$, et al. Efficiency of antenatal care and childbirth services in selected primary health care facilities in rural Tanzania: a cross-sectional study. BMC Health Serv Res. 2014;14:96.

19. Hitimana R, Lindholm L, Krantz G, Nzayirambaho M, Pulkki-Brannstrom AM. Cost of antenatal care for the health sector and for households in Rwanda. BMC Health Serv Res. 2018;18(1):262.

20. Hitimana R, Lindholm L, Mogren I, Krantz G, Nzayirambaho M, Sengoma JS, et al. Incremental cost and health gains of the 2016 WHO antenatal care recommendations for Rwanda: results from expert elicitation. Health research policy and systems. 2019;17(1):36.

21. NBS Tanzania, Chief government statistician Zanzibar. National Population Projections. 2018

22. Drummond MF, Sculpher MJ, Slaxton K, Stoddart GL, Torrance GW. Methods for the economic evaluation of health care programmes. 4th ed. New York: Oxford University Press; 2015.

23. Masinde A, Gumodoka B, Kilonzo A, Mshana SE. Prevalence of urinary tract infection among pregnant women at Bugando medical centre, Mwanza, Tanzania. Tanzan J Health Res. 2009;1 1(3):154-9.

24. Fernandes S, Sicuri E, Halimatou D, Akazili J, Boiang K, Chandramohan D, et al. Cost effectiveness of intermittent screening followed by treatment versus intermittent preventive treatment during pregnancy in West Africa: analysis and modelling of results from a non-inferiority trial. Malar J. 2016;15(1):493.
25. World Health Organization. Cost effectiveness and strategic planning (WHO-CHOICE): prices and useful lives of tradable capital goods. Geneva: World Health Organization; 2008.

26. Mathewos B, Owen H, Sitrin D, Cousens S, Degefie T, Wall S, et al. Community-based interventions for newborns in Ethiopia (COMBINE): cost-effectiveness analysis. Health Policy Plan. 2017;32(suppl_1):i21-32.

27. UNAIDS. Costing guidelines for HIV prevention strategies. Geneva: UNAIDS; 2000. https://data.unaids.org/publications/irc-pub05/jc412costguidel_en.pdf.

28. Bank of Tanzania. Financial markets, interbanking foreign exchange market (IFEM) summaries. Dar Es Salaam. https://www.bot.go.tz/.

29. von Both C, Flessa S, Makuwani A, Mpembeni R, Jahn A. How much time do health services spend on antenatal care? Implications for the introduction of the focused antenatal care model in Tanzania. BMC Pregnancy Childbirth. 2006;6:22.

30. Bintabara D, Nakamura K, Ntwenya J, Seino K, Mpondo BCT. Adherence to standards of first-visit antenatal care among providers: a stratified analysis of Tanzanian facility-based survey for improving quality of antenatal care. PloS One. 2019;14(5): e0216520.

31. Memirie ST, Tolla MT, Desalegn D, Hailemariam M, Norheim OF, Verguet $\mathrm{S}$, et al. A cost-effectiveness analysis of maternal and neonatal health interventions in Ethiopia. Health Policy Plan. 2019;34(4):289-97.

32. Goldenberg RL, Nathan RO, Swanson D, Saleem S, Mirza W, Esamai F, et al. Routine antenatal ultrasound in low- and middle-income countries: first look - a cluster randomised trial. BJOG Int J Obstet Gynaecol. 2018;125(12):1591-9.

\section{Publisher's Note}

Springer Nature remains neutral with regard to jurisdictional claims in published maps and institutional affiliations.

Ready to submit your research? Choose BMC and benefit from:

- fast, convenient online submission

- thorough peer review by experienced researchers in your field

- rapid publication on acceptance

- support for research data, including large and complex data types

- gold Open Access which fosters wider collaboration and increased citations

- maximum visibility for your research: over $100 \mathrm{M}$ website views per year

At BMC, research is always in progress.

Learn more biomedcentral.com/submissions 